\title{
Minimum Value Derivation Technique
}

National Cancer Institute

\section{Source}

National Cancer Institute. Minimum Value Derivation Technique. NCI Thesaurus. Code C82867.

A data derivation technique used to calculate a subject's minimum value over a defined set of records. 